\title{
Tacrolimus for the Treatment of Ulcerative Colitis
}

\author{
Katsuyoshi Matsuoka, Eiko Saito, Toshimitsu Fujii, Kento Takenaka, Maiko Kimura, Masakazu Nagahori, \\ Kazuo Ohtsuka, Mamoru Watanabe \\ Department of Gastroenterology and Hepatology, Tokyo Medical and Dental University, Tokyo, Japan
}

Tacrolimus is a calcineurin inhibitor used for the treatment of corticosteroid-refractory ulcerative colitis (UC). Two randomized controlled trials and a number of retrospective studies have assessed the therapeutic effect of tacrolimus in UC patients. These studies showed that tacrolimus has excellent short-term efficacy in corticosteroid-refractory patients, with the rates of clinical response ranging from $61 \%$ to $96 \%$. However, the long-term prognosis of patients treated with tacrolimus is disappointing, and almost 50\% of patients eventually underwent colectomy in long-term follow-up. Tacrolimus can achieve mucosal healing in $40-50 \%$ of patients, and this is associated with a favorable long-term prognosis. Anti-tumor necrosis factor (TNF)- $\alpha$ antibodies are another therapeutic option in corticosteroid-refractory patients. A prospective head-to-head comparative study of tacrolimus and infliximab is currently being performed to determine which treatment is more effective in corticosteroid-refractory patients. Several retrospective studies have demonstrated that switching between tacrolimus and anti-TNF- $\alpha$ antibody therapy was effective in patients who were refractory to one of the treatments. Most adverse events of tacrolimus are mild; however, opportunistic infections, especially pneumocystis pneumonia, are the most important adverse events, and these should be carefully considered during treatment. Several issues on tacrolimus treatment in UC patients remain unsolved (e.g., use of tacrolimus as remission maintenance therapy). Further controlled studies are needed to optimize the use of tacrolimus for the treatment of UC. (Intest Res 2015;13:219-226)

Key Words: Tacrolimus; Colitis, ulcerative; Cyclosporine; Calcineurin inhibitors

\section{INTRODUCTION}

Ulcerative colitis (UC) is a chronic disease with a relapseremitting course. Corticosteroids are the first choice in patients with moderate-to-severe disease activity. Although corticosteroids are an effective treatment option, approximately $40 \%$ of patients are resistant to or dependent on corticosteroids.

\footnotetext{
Received May 4, 2014. Revised May 6, 2014. Accepted May 6, 2014. Correspondence to Mamoru Watanabe, Department of Gastroenterology and Hepatology, Tokyo Medical and Dental University, 1-5-45 Yushima, Bunkyo-ku, Tokyo 113-8519, Japan. Tel: +81-3-5803-5877, Fax: +81-35803-0268, E-mail: mamoru.gast@tmd.ac.jp

Financial support: This study was supported in part by Grants-in-Aid from the Japanese Ministry of Education, Culture, Sports, Science and Technology: Health and Labour, Sciences Research Grants for research on intractable diseases from the Ministry of Health, Labour and Welfare of Japan. Conflict of interest: None.
}

The treatment of corticosteroid-refractory UC is challenging, and colectomy is often indicated in these patients. Cyclosporine, a calcineurin inhibitor, has been used as a salvage therapy in corticosteroid-refractory patients. Lichtiger et al. reported a randomized controlled trial on the use of cyclosporine in acute severe UC. ${ }^{1}$ In this trial, 20 corticosteroid-refractory severe UC patients were randomly assigned to receive intravenous cyclosporine infusion at $4 \mathrm{mg} / \mathrm{kg}$ daily or placebo. The response rate at 7 days was $82 \%(9 / 11)$ in the cyclosporine group compared to $0 \%(0 / 9)$ in the placebo group. Intravenous cyclosporine is usually followed by oral cyclosporine, but intestinal absorption of cyclosporine is poor and unstable, and therefore, it is difficult to achieve optimal trough levels with oral cyclosporine.

Tacrolimus is a macrolide antibiotic, isolated from the soil bacterium Streptomyces tsukubaensis. It possesses potent immunosuppressive properties and has been used to prevent organ rejection after allogeneic organ transplantation

\footnotetext{
๑ Copyright 2015. Korean Association for the Study of Intestinal Diseases. All rights reserved.

This is an Open Access article distributed under the terms of the Creative Commons Attribution Non-Commercial License (http://creativecommons.org/licenses/by-nc/4.0)

which permits unrestricted non-commercial use, distribution, and reproduction in any medium, provided the original work is properly cited.
} 
or graft-versus-host disease after hematopoietic stem cell transplantation. The absorption of tacrolimus through the intestine is much better than that of cyclosporine. Considering these properties of tacrolimus, it is commonly used for the treatment of corticosteroid-refractory UC instead of cyclosporine. In Japan, tacrolimus was approved for clinical use in corticosteroid-refractory UC patients in 2010 and has been incorporated in the management of refractory UC. Here, we review the literature on the use of tacrolimus in UC patients.

\section{MECHANISM OF ACTION}

Tacrolimus mainly exerts its immunosuppressive action by inhibiting T-cell activation and proliferation. Upon T-cell receptor activation by antigen-presenting cells, intracellular calcium concentrations increase, leading to activation of calcineurin, a calcium and calmodulin-dependent phosphatase. The transcription factor, nuclear factor of activated Tcells (NFAT) is dephosphorylated by calcineurin, and then, it moves to the nucleus and induces transcription of interleukin-2 and other related genes. interleukin-2 is essential for T-cell activation and proliferation. Tacrolimus binds to the immunophilin FK binding protein (FKBP) and forms a complex, which binds to calcineurin and inhibits its activity. The immunosuppressive properties of tacrolimus have been reported to be 100 times greater than those of cyclosporine both in vivo and in vitro., ${ }^{2,3}$

\section{REMISSION INDUCTION}

The first case series to assess the efficacy of tacrolimus for IBD was reported in 1998. ${ }^{4}$ This study included 11 corticosteroid-refractory patients (6 with UC, 2 with indeterminate colitis, 2 with $\mathrm{CD}$, and 1 with pouchitis). All patients were treated with intravenous tacrolimus for 7-10 days, followed by oral tacrolimus treatment. Of the 11 patients, 7 (63.6\%) achieved remission.

The first randomized double-blind controlled trial on the use of oral tacrolimus in UC patients was reported by Ogata et al. in 2006. ${ }^{5}$ In this study, 60 corticosteroid-refractory UC patients were randomly assigned to receive oral tacrolimus at high serum trough $(10-15 \mathrm{ng} / \mathrm{mL} ; \mathrm{n}=19)$ or low serum trough $(5-10 \mathrm{ng} / \mathrm{mL} ; \mathrm{n}=21)$ levels, or placebo $(\mathrm{n}=20)$. At 2 weeks after treatment, the clinical response rates were $68.4 \%$ and $38.1 \%$ in the high trough and low trough groups, respectively, and $10.0 \%$ in the placebo group. Ogata et al. further examined the efficacy of oral tacrolimus in a large randomized controlled trial that included 62 patients with corticosteroidrefractory, moderate-to-severe UC. ${ }^{6}$ In this study, the serum trough levels were fixed at $10-15 \mathrm{ng} / \mathrm{mL}$ in the tacrolimus group. A result similar to that of the first study was obtained, with clinical response rates of $50.0 \%$ in the tacrolimus group and $\mathbf{1 3 . 3 \%}$ in the placebo group. Presently, these studies are the only controlled trials on the efficacy of tacrolimus in UC patients.

Many retrospective cohort studies have examined the efficacy of tacrolimus in UC patients in real-world settings ${ }^{4-21}$ (Table 1). The largest study was reported from Germany, and it included 130 patients from 3 institutions. ${ }^{15}$ In this study, clinical remission was achieved in 94 patients (72\%). Furthermore, the authors reported that concomitant use of thiopurine significantly increased the rate of remission. Miyoshi et al. reported 51 patients who were treated with oral tacrolimus. In this study, the clinical response rate at 3 months after treatment was $62.7 \%(32 / 53 ; 20$ patients $(39.2 \%)$ in remission, 12 patients (23.5\%) with improvement). ${ }^{22}$

Several retrospective studies have shown the efficacy of tacrolimus in pediatric patients. A study that included 46 pediatric patients with corticosteroid-refractory colitis showed that $93 \%$ of patients avoided immediate colectomy with tacrolimus. ${ }^{23,24}$ Ziring et al. reported the outcomes of 18 consecutive pediatric patients who were treated with oral tacrolimus. Of the 18 patients, 17 (94.4\%) responded to treatment. ${ }^{25}$

Taken together, these findings suggest that tacrolimus is an effective treatment for remission induction in corticosteroidrefractory UC patients, although evidence from controlled studies is limited.

\section{LONG-TERM PROGNOSIS}

Despite the high response rates in remission induction with tacrolimus, long-term prognosis after tacrolimus treatment is disappointing. Miyoshi et al. analyzed 51 patients who were treated with tacrolimus and reported that the relapse-free survival rates were $73.0 \%$ at 6 months, $49.9 \%$ at 1 year, and $37.8 \%$ at 2 years after treatment. ${ }^{22}$ Additionally, a retrospective study from 3 French referral centers demonstrated that only $27 \%$ of patients had clinical remission at 54 weeks after treatment. ${ }^{26}$ Furthermore, the rates of long-term colectomy-free survival have been reported to be $56.5 \%$ at 43.8 months, ${ }^{10} 59.3 \%$ at 2 years, ${ }^{16}$ and $62.3 \%$ at 65 months after treatment. ${ }^{12}$ These findings demonstrate that immediate colectomy may be avoided with tacrolimus; however, about $50.0 \%$ of patients eventually required colectomy in long- 


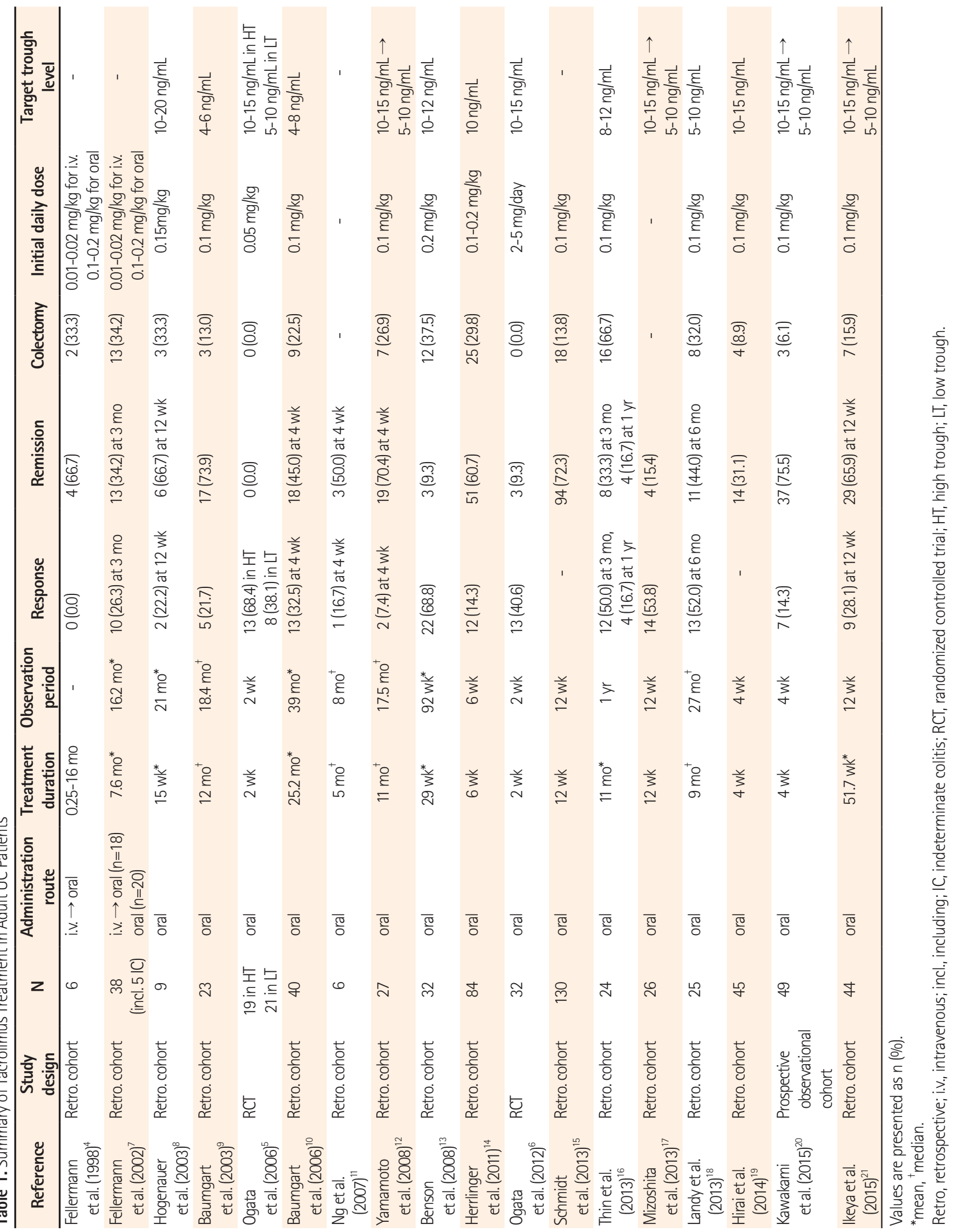


term follow-up.

A few uncontrolled studies suggested the effect of tacrolimus on remission maintenance. A study by Baumgart et al. assessed the long-term efficacy of oral low-dose tacrolimus in 40 UC patients. Of these 40 patients, 31 (77.5\%) maintained remission during a mean treatment duration of 25.2 months at serum trough levels of $4-8 \mathrm{ng} / \mathrm{mL} .^{10}$ A retrospective analysis by Yamamoto et al. showed that the longterm relapse-free survival rates of patients administered tacrolimus as remission induction therapy $(n=24)$ were comparable to those of patients administered thiopurine $(\mathrm{n}=34) .{ }^{27}$ These findings suggest that tacrolimus can be used as remission maintenance therapy. Randomized controlled studies are necessary to show the effects of long-term tacrolimus administration as remission maintenance therapy in UC patients. Additionally, studies to determine the optimal duration and trough levels for remission maintenance are required.

\section{MUCOSAL HEALING}

Mucosal healing has been an emerging therapeutic target in UC treatment. In the study by Ogata et al. in 2012, the rate of mucosal healing at 12 weeks after treatment was $43.8 \%$ $(14 / 32)$ in the tacrolimus group and $13.3 \%(4 / 30)$ in the placebo group. ${ }^{6}$ Miyoshi et al. reported the endoscopic results of 36 patients who received oral tacrolimus treatment and underwent colonoscopy after 3 months of treatment. Of these 36 patients, 12 (33.3\%) and $10(27.8 \%)$ showed Mayo endoscopic scores of 0 and 1, respectively. ${ }^{22}$ Furthermore, patients with a Mayo endoscopic score of 0 or 1 had a significantly better medium-to-long term prognosis compared to that in those with a score of 3 or 4 . Additionally, Ikeya et al. reported that the rate of mucosal healing was $43.8 \%$ (14/32) in patients treated with tacrolimus and that mucosal healing was associated with a favorable prognosis. ${ }^{21}$ These findings confirm that mucosal healing can be achieved with tacrolimus and may be used as a therapeutic target of this treatment.

\section{TACROLIMUS VERSUS ANTI-TUMOR NECROSIS FACTOR (TNF)-ALPHA ANTIBODY THERAPY}

Anti-TNF- $\alpha$ antibody therapy is an option for the treatment of corticosteroid-refractory UC. ${ }^{28,29}$ Thus far, no direct comparative studies have assessed the efficacy of tacrolimus and anti-TNF- $\alpha$ antibody therapy in UC patients. A randomized, prospective head-to-head comparative study of tacrolimus and infliximab in corticosteroid-refractory UC patients is being performed in Japan.

A recent randomized controlled study demonstrated that infliximab and cyclosporine, another calcineurin inhibitor, were equally effective for remission induction in corticosteroid-refractory acute severe UC patients. ${ }^{30}$ Additionally, several retrospective studies have shown a similar efficacy between infliximab and cyclosporine for remission induction; however, long-term prognosis was more favorable in patients treated with infliximab than in those treated with cyclosporine. ${ }^{31-34}$ A recent retrospective study assessed short-term outcomes in patients with severe UC treated with tacrolimus $(\mathrm{n}=22)$ or infliximab $(\mathrm{n}=7) .^{35}$ The rates of clinical remission were $63.6 \%$ and $71.4 \%$ in the tacrolimus and infliximab groups, respectively.

These findings suggest that the therapeutic effect of calcineurin inhibitors is comparable to that of infliximab in terms of remission induction. However, the mechanisms of action of these drugs are completely different. Therefore, it is important to select an appropriate therapy in each patient. Additionally, the optimization of remission maintenance therapy is challenging after calcineurin inhibitor therapy.

\section{SWITCHING BETWEEN TACROLIMUS AND ANTI-TNF-ALPHA ANTIBODY THERAPY}

Three studies have used infliximab as rescue therapy in patients who were refractory to tacrolimus, and the shortterm response rates were $25.0 \%(6 / 24),{ }^{36} 46.2 \%(6 / 13),{ }^{37}$ and $50.0 \%(6 / 12)$ in these studies. ${ }^{38}$ Yamamoto et al. further showed that the rate of colectomy-free survival was $58.3 \%$ at 41.4 months after treatment. ${ }^{38}$ Conversely, Boschetti et al. evaluated the efficacy of tacrolimus in 30 patients who were refractory to anti-TNF- $\alpha$ antibody therapy ${ }^{26}$ In this study, clinical response was observed in 21 patients $(70 \%)$ at 4 weeks after treatment, with 14 patients (47\%) in clinical remission, and 8 patients (27\%) maintained clinical remission at 52 weeks after treatment. These findings suggest that switching between tacrolimus and anti-TNF- $\alpha$ antibody therapy may be an effective therapeutic option in selected patients. However, evidence on long-term prognosis is scarce.

Attention should be paid to the risk of opportunistic infections when switching between 2 immunosuppressive agents. ${ }^{39}$ Additionally, there is a concern that the use of multiple immunosuppressive agents increases perioperative complications. Saito et al. reported that the frequency of postoperative complications did not increase in patients who underwent colectomy after receiving multiple immunosuppressive agents compared to that in patients who under- 
went colectomy after receiving only 1 immunosuppressive agent. ${ }^{40}$ The benefits and risks should be carefully considered when switching between a calcineurin inhibitor and anti-TNF- $\alpha$ antibody therapy.

\section{BLOOD TROUGH LEVELS}

The therapeutic effect of tacrolimus appears to depend on the trough level. In the study by Ogata et al. in 2006, the clinical response rate was higher in the high trough (10-15 ng/ $\mathrm{mL}$ ) group than in the low trough $(5-10 \mathrm{ng} / \mathrm{mL})$ group. ${ }^{5} \mathrm{Ad}-$ ditionally, the study by Ogata et al. in 2012 showed that continuation of tacrolimus at a low trough level $(5-10 \mathrm{ng} / \mathrm{mL})$ until 12 weeks increased the rate of mucosal healing from $66.7 \%(14 / 21)$ to $85.7 \%(18 / 21)$ and that of clinical remission from $14.3 \%$ (3/21) to $28.6 \%(6 / 21){ }^{6}$ Based on these findings, the standard target trough level in Japan is $10-15 \mathrm{ng} / \mathrm{mL}$ for 2 weeks, followed by $5-10 \mathrm{ng} / \mathrm{mL}$ for 12 weeks. However, for long-term use of tacrolimus, the optimal trough level has not yet been determined.

Several factors affect the blood trough levels of tacrolimus. First, fasting affects the pharmacokinetics of tacrolimus. $\mathrm{C}_{\max }$ is almost 4 times higher in a fasting condition than in a fed condition. ${ }^{41}$ Second, genetic backgrounds can affect the blood trough levels of tacrolimus. Tacrolimus is metabolized predominantly by cytochrome P-450 (CYP) 3A4 and $3 A 5$ enzymes, and is a substrate for the drug efflux pump Pglycoprotein encoded by the $A B C B 1$ gene. A German study reported that the short-term response to tacrolimus was associated with homozygous variants for 1 of the $3 A B C B 1$ alleles, but not with $C Y P 3 A 5$ polymorphisms. ${ }^{14}$ Interestingly, a Japanese study reported opposite results. Hirai et al. examined the expression of CYP3A5 in 45 patients treated with tacrolimus. ${ }^{19}$ Of these 45 patients, 24 (53.3\%) were CYP3A5 expressers and 21 (46.7\%) were non-expressers. The trough levels at 2-5 days after treatment were significantly higher in the CYP3A5 expressers than in the non-expressers. This rapid increase in trough levels was associated with a higher rate of remission in the non-expressers (47.6\%) than in the expressers (16.7\%). ABCB1 gene polymorphisms were not associated with trough levels. These findings suggest a genetic difference between Asian and Caucasian populations in tacrolimus metabolism.

\section{ROUTE OF ADMINISTRATION}

Both oral and intravenous formulas are available in tacrolimus treatment. The oral formula is often used for the treat- ment of UC, because tacrolimus is efficiently absorbed from the intestine.

Fellermann et al. compared intravenous administration of tacrolimus with oral administration in 38 patients with colitis (33 with UC and 5 with indeterminate colitis). ${ }^{7}$ In this study, tacrolimus was administered intravenously at a dose of $0.01-0.02 \mathrm{mg} / \mathrm{kg}$ in 18 patients for up to 14 days, followed by oral administration. Additionally, it was administered orally at a dose of $0.1-0.2 \mathrm{mg} / \mathrm{kg}$ in 20 patients. The efficacy was similar between the intravenous and oral groups in terms of the rates of response, remission, and colectomy. Additionally, blood tacrolimus levels were comparable between the 2 groups. In our experience, response rates are similar in patients treated with tacrolimus intravenously and those treated orally. However, target blood levels can be achieved within a few days with intravenous infusion, while it takes 4-5 days to reach target blood trough levels with oral administration (unpublished data). Thus, the therapeutic response can be determined earlier in intravenously treated patients than in orally treated patients, and this earlier decision can be critical in severely ill patients.

Rectal administration of tacrolimus may be effective in patients with left-sided colitis or proctitis, because tacrolimus ointment is used to treat atopic dermatitis. A study examined the efficacy of tacrolimus enema or suppository in distal colitis. ${ }^{42}$ The enema and suppository contained $2-4 \mathrm{mg}$ and $2 \mathrm{mg}$ of tacrolimus, respectively. After treatment for 4 weeks, 13 of 19 patients (68.4\%) showed clinical improvement. Additionally, a small prospective study demonstrated that 6 of 8 patients with refractory distal colitis achieved remission with rectal tacrolimus administration. ${ }^{43}$ Uchino et al. used tacrolimus enema in 10 patients with antibiotic-refractory pouchitis. ${ }^{44}$ In this study, clinical symptoms improved in 9 patients $(90.0 \%)$ after 8 weeks of treatment. Based on these findings, rectal tacrolimus treatment may be effective and should be examined in future randomized controlled studies.

\section{ADVERSE EVENTS}

A systematic review examining tacrolimus use in UC patients reported that the most frequently observed adverse events were neurotoxicity, including tremor and headache, followed by gastrointestinal disorders, nephrotoxicity, and metabolic disorders. ${ }^{45}$ Most of the adverse events were mild, and could be attenuated by reducing the dose of tacrolimus. Opportunistic infections, especially pneumocystis pneumonia, are the most important adverse events, and these should be carefully considered during tacrolimus treatment. ${ }^{46}$ Pro- 
phylaxis for pneumocystis pneumonia and its close monitoring should be considered in patients treated with tacrolimus.

Safety data on the long-term administration of tacrolimus in UC patients are limited. Baumgart et al. reported on 53 IBD patients ( 40 with UC, 11 with CD, and 2 with pouchitis) who were treated with tacrolimus for a mean of 25.2 months. ${ }^{10}$ In this study, the most common adverse events were tremor and paresthesia $(n=5,9.4 \%)$, followed by a temporal rise in serum creatinine levels $(n=4,7.6 \%)$. Nephrotoxicity may be a limiting factor for the long-term use of tacrolimus.

\section{CONCLUSIONS}

Tacrolimus shows excellent efficacy for remission induction in corticosteroid-refractory UC patients. However, several questions remain unanswered: (1) Can tacrolimus be used as maintenance therapy? (2) What is the optimal trough level for long-term use? (3) What is the optimal administration route? (4) How to determine whether to administer tacrolimus or anti-TNF- $\alpha$ antibody therapy? (5) Is combination therapy of low-dose tacrolimus and infliximab, which has been reported to be effective in rheumatoid arthritis patients, feasible in UC patients? More controlled studies are needed to answer these clinical questions regarding tacrolimus and optimize its use in the treatment of UC. Furthermore, the identification of predictive factors or development of biomarkers to predict therapeutic responses to tacrolimus is needed. Tacrolimus is used primarily in Japan among the Asian countries, ${ }^{47}$ and it can become an indispensable treatment option for corticosteroid-refractory UC globally with further optimization of its use.

\section{REFERENCES}

1. Lichtiger S, Present DH, Kornbluth A, et al. Cyclosporine in severe ulcerative colitis refractory to steroid therapy. N Engl J Med 1994;330:1841-1845.

2. Kino T, Hatanaka H, Miyata S, et al. FK-506, a novel immunosuppressant isolated from a Streptomyces. II. Immunosuppressive effect of FK-506 in vitro. J Antibiot (Tokyo) 1987;40:12561265 .

3. Hirano T, Akashi T, Kido T, Oka K, Shiratori T, Miyaoka M. Immunosuppressant pharmacodynamics on peripheral-blood mononuclear cells from patients with ulcerative colitis. Int Immunopharmacol 2002;2:1055-1063.
4. Fellermann K, Ludwig D, Stahl M, David-Walek T, Stange EF. Steroid-unresponsive acute attacks of inflammatory bowel disease: immunomodulation by tacrolimus (FK506). Am J Gastroenterol 1998;93:1860-1866.

5. Ogata H, Matsui T, Nakamura M, et al. A randomised dose finding study of oral tacrolimus (FK506) therapy in refractory ulcerative colitis. Gut 2006;55:1255-1262.

6. Ogata H, Kato J, Hirai F, et al. Double-blind, placebo-controlled trial of oral tacrolimus (FK506) in the management of hospitalized patients with steroid-refractory ulcerative colitis. Inflamm Bowel Dis 2012;18:803-808.

7. Fellermann K, Tanko Z, Herrlinger KR, et al. Response of refractory colitis to intravenous or oral tacrolimus (FK506). Inflamm Bowel Dis 2002;8:317-324.

8. Högenauer C, Wenzl HH, Hinterleitner TA, Petritsch W. Effect of oral tacrolimus (FK 506) on steroid-refractory moderate/ severe ulcerative colitis. Aliment Pharmacol Ther 2003;18:415423.

9. Baumgart DC, Wiedenmann B, Dignass AU. Rescue therapy with tacrolimus is effective in patients with severe and refractory inflammatory bowel disease. Aliment Pharmacol Ther 2003;17:1273-1281.

10. Baumgart DC, Pintoffl JP, Sturm A, Wiedenmann B, Dignass AU. Tacrolimus is safe and effective in patients with severe steroidrefractory or steroid-dependent inflammatory bowel diseasea long-term follow-up. Am J Gastroenterol 2006;101:1048-1056.

11. Ng SC, Arebi N, Kamm MA. Medium-term results of oral tacrolimus treatment in refractory inflammatory bowel disease. Inflamm Bowel Dis 2007;13:129-134.

12. Yamamoto S, Nakase H, Mikami S, et al. Long-term effect of tacrolimus therapy in patients with refractory ulcerative colitis. Aliment Pharmacol Ther 2008;28:589-597.

13. Benson A, Barrett T, Sparberg M, Buchman AL. Efficacy and safety of tacrolimus in refractory ulcerative colitis and Crohn's disease: a single-center experience. Inflamm Bowel Dis 2008;14:7-12.

14. Herrlinger KR, Koc $\mathrm{H}$, Winter S, et al. $A B C B 1$ single-nucleotide polymorphisms determine tacrolimus response in patients with ulcerative colitis. Clin Pharmacol Ther 2011;89:422-428.

15. Schmidt KJ, Herrlinger KR, Emmrich J, et al. Short-term efficacy of tacrolimus in steroid-refractory ulcerative colitis - experience in 130 patients. Aliment Pharmacol Ther 2013;37:129-136.

16. Thin LW, Murray K, Lawrance IC. Oral tacrolimus for the treatment of refractory inflammatory bowel disease in the biologic era. Inflamm Bowel Dis 2013;19:1490-1498. 
17. Mizoshita T, Tanida S, Tsukamoto H, et al. Colon mucosa exhibits loss of ectopic MUC5AC expression in patients with ulcerative colitis treated with oral tacrolimus. ISRN Gastroenterol 2013;2013:304894.

18. Landy J, Wahed M, Peake ST, et al. Oral tacrolimus as maintenance therapy for refractory ulcerative colitis-an analysis of outcomes in two London tertiary centres. J Crohns Colitis 2013;7:e516-e521.

19. Hirai F, Takatsu N, Yano Y, et al. Impact of CYP3A5 genetic polymorphisms on the pharmacokinetics and short-term remission in patients with ulcerative colitis treated with tacrolimus. J Gastroenterol Hepatol 2014;29:60-66.

20. Kawakami K, Inoue T, Murano M, et al. Effects of oral tacrolimus as a rapid induction therapy in ulcerative colitis. World J Gastroenterol 2015;21:1880-1886.

21. Ikeya K, Sugimoto K, Kawasaki S, et al. Tacrolimus for remission induction in ulcerative colitis: Mayo endoscopic subscore 0 and 1 predict long-term prognosis. Dig Liver Dis 2015;47:365-371.

22. Miyoshi J, Matsuoka K, Inoue N, et al. Mucosal healing with oral tacrolimus is associated with favorable medium- and long-term prognosis in steroid-refractory/dependent ulcerative colitis patients. J Crohns Colitis 2013;7:e609-e614.

23. Watson S, Pensabene L, Mitchell P, Bousvaros A. Outcomes and adverse events in children and young adults undergoing tacrolimus therapy for steroid-refractory colitis. Inflamm Bowel Dis 2011;17:22-29.

24. Bousvaros A, Kirschner BS, Werlin SL, et al. Oral tacrolimus treatment of severe colitis in children. J Pediatr 2000;137:794799.

25. Ziring DA, Wu SS, Mow WS, Martín MG, Mehra M, Ament ME. Oral tacrolimus for steroid-dependent and steroid-resistant ulcerative colitis in children. J Pediatr Gastroenterol Nutr 2007;45:306-311.

26. Boschetti G, Nancey S, Moussata D, et al. Tacrolimus induction followed by maintenance monotherapy is useful in selected patients with moderate-to-severe ulcerative colitis refractory to prior treatment. Dig Liver Dis 2014;46:875-880.

27. Yamamoto S, Nakase H, Matsuura M, Masuda S, Inui K, Chiba T. Tacrolimus therapy as an alternative to thiopurines for maintaining remission in patients with refractory ulcerative colitis. J Clin Gastroenterol 2011;45:526-530.

28. Rutgeerts P, Sandborn WJ, Feagan BG, et al. Infliximab for induction and maintenance therapy for ulcerative colitis. $\mathrm{N}$ Engl J Med 2005;353:2462-2476.

29. Reinisch W, Sandborn WJ, Hommes DW, et al. Adalimumab for induction of clinical remission in moderately to severely active ulcerative colitis: results of a randomised controlled trial. Gut 2011;60:780-787.
30. Laharie D, Bourreille A, Branche J, et al. Ciclosporin versus infliximab in patients with severe ulcerative colitis refractory to intravenous steroids: a parallel, open-label randomised controlled trial. Lancet 2012;380:1909-1915.

31. Naves JE, Llaó J, Ruiz-Cerulla A, et al. Long-term comparative efficacy of cyclosporine- or infliximab-based strategies for the management of steroid-refractory ulcerative colitis attacks. Inflamm Bowel Dis 2014;20:1375-1381.

32. Croft A, Walsh A, Doecke J, Cooley R, Howlett M, RadfordSmith G. Outcomes of salvage therapy for steroid-refractory acute severe ulcerative colitis: ciclosporin vs. infliximab. Aliment Pharmacol Ther 2013;38:294-302.

33. Dean KE, Hikaka J, Huakau JT, Walmsley RS. Infliximab or cyclosporine for acute severe ulcerative colitis: a retrospective analysis. J Gastroenterol Hepatol 2012;27:487-492.

34. Mocciaro F, Renna S, Orlando A, et al. Cyclosporine or infliximab as rescue therapy in severe refractory ulcerative colitis: early and long-term data from a retrospective observational study. J Crohns Colitis 2012;6:681-686.

35. Minami N, Yoshino T, Matsuura M, et al. Tacrolimus or infliximab for severe ulcerative colitis: short-term and long-term data from a retrospective observational study. BMJ Open Gastro doi:10.1136/bmjgast-2014-000021. Published online 20 February 2015 .

36. Herrlinger KR, Barthel DN, Schmidt KJ, et al. Infliximab as rescue medication for patients with severe ulcerative/indeterminate colitis refractory to tacrolimus. Aliment Pharmacol Ther 2010;31:1036-1041.

37. Tsukamoto H, Tanida S, Mizoshita T, et al. Infliximab salvage therapy for patients with ulcerative colitis who failed to respond to tacrolimus. Eur J Gastroenterol Hepatol 2013;25:714-718.

38. Yamamoto S, Nakase H, Matsuura M, et al. Efficacy and safety of infliximab as rescue therapy for ulcerative colitis refractory to tacrolimus. J Gastroenterol Hepatol 2010;25:886-891.

39. Leblanc S, Allez M, Seksik P, et al. Successive treatment with cyclosporine and infliximab in steroid-refractory ulcerative colitis. Am J Gastroenterol 2011;106:771-777.

40. Saito E, Nagahori M, Fujii T, Ohtsuka K, Watanabe M. Efficacy of salvage therapy and its effect on operative outcomes in patients with ulcerative colitis. Digestion 2014;89:55-60.

41. Lainesse A, Hussain S, Monif T, et al. Bioequivalence studies of tacrolimus capsule under fasting and fed conditions in healthy male and female subjects. Arzneimittelforschung 2008;58:242247.

42. van Dieren JM, van Bodegraven AA, Kuipers EJ, et al. Local application of tacrolimus in distal colitis: feasible and safe. Inflamm Bowel Dis 2009;15:193-198. 
43. Lawrance IC, Copeland TS. Rectal tacrolimus in the treatment of resistant ulcerative proctitis. Aliment Pharmacol Ther 2008;28:1214-1220.

44. Uchino M, Ikeuchi H, Matsuoka H, et al. Topical tacrolimus therapy for antibiotic-refractory pouchitis. Dis Colon Rectum 2013;56:1166-1173.

45. Gonzalez-Lama Y, Gisbert JP, Mate J. The role of tacrolimus in inflammatory bowel disease: a systematic review. Dig Dis Sci 2006;51:1833-1840.
46. Escher M, Stange EF, Herrlinger KR. Two cases of fatal Pneumocystis jirovecii pneumonia as a complication of tacrolimus therapy in ulcerative colitis-a need for prophylaxis. J Crohns Colitis 2010;4:606-609.

47. Hida N, Nakamura S, Hahm KB, et al. A questionnaire-based survey on the diagnosis and management of inflammatory bowel disease in East Asian countries in 2012. Digestion 2014;89:88-103. 